\title{
UNIVERSITY OF MIAMI RADIOCARBON DATES XI
}

\section{PIEPGRAS, M M CALVERT, and J J STIPP}

Department of Geology, University of Miami, Coral Gables, Florida 33124

The following dates are a partial list of geologic samples dated since December 1976. The method used is described by (Stipp et al, 1976). Ages were calculated using a half-life of 5568 years. Errors reported are one standard deviation and include only the counting errors on the unknown sample, background and modern standard. There have been no corrections made on these dates. Sample descriptions and comments were written, based on information supplied by the submitters.

\section{SAMPLE DESCRIPTIONS}

\section{A. United States}

\section{North Captiva Island Series}

Shell samples hand coll along E-W transect on North Captiva I., Florida $\left(26^{\circ} 32^{\prime}\right.$ to $36^{\prime} \mathrm{N}, 82^{\circ} 10^{\prime}$ to $\left.15^{\prime} \mathrm{W}\right)$. Coll from ca $0.5 \mathrm{~m}$ below island surface. Dated to establish method of barrier island formation. Coll and subm 1977 by T Missimer, Cape Coral, Florida and C Snively, Univ Miami.

UM-1069. Ia

UM-1070a. 1 b

UM-1070b. $1 \mathrm{lb}$

Duplicate run of UM-1070a.

UM-1071. 2

UM-1072. 3

UM-1073. 4

UM-1074. 9

UM-1075. 10
$104.0 \pm 0.9 \%$ modern

$$
3825 \pm 105
$$$$
2450 \pm 80
$$

$$
1400 \pm 75
$$

$1850 \pm 75$

$920 \pm 85$

$435 \pm 65$

$1730 \pm 85$

\section{Everglades Tree Island series}

Peat from piston core in Everglades tree-island, small Persea type, in Conservation Area I, Everglades, Florida $\left(26^{\circ} 26^{\prime} 55^{\prime \prime} \mathrm{N}, 80^{\circ} 17^{\prime} 10^{\prime \prime}\right.$ W). Continuation of study on tree-island formation (R, 1976, v. 18, p 375; v 19, p 121-122). Coll and subm 1976 by D Piepgras.

General Comment (DP): this core was taken $9.6 \mathrm{~km} \mathrm{~N}$ of Core $20(\mathrm{R}$, 1976 , v 19, p 121-122) on same island. Results support floating island theory and indicate that subsequent island growth is affected by Everglades drainage patterns (Davis, 1943). Core 20 showed no reversals.

\section{UM-1106. $70 \mathrm{~cm}$}

$290 \pm 80$

Wood. 
UM-1107. 81 to $91 \mathrm{~cm}$

$$
410 \pm 70
$$

UM-1108. 141 to $147 \mathrm{~cm}$

$1300 \pm 135$

UM-1109. 150 to $156 \mathrm{~cm}$

$1710 \pm 110$

UM-1110. 158 to $164 \mathrm{~cm}$

$1260 \pm 70$

UM-1111. 175 to $181 \mathrm{~cm}$

$1900 \pm 85$

UM-1112. 183 to $187 \mathrm{~cm}$

$2620 \pm 115$

UM-1113. 189 to $195 \mathrm{~cm}$

$1805 \pm 60$

UM-1114. 195 to $200 \mathrm{~cm}$

$1850 \pm 95$

UM-1115. 200 to $206 \mathrm{~cm}$

$2295 \pm 60$

UM-1116. 206 to $213 \mathrm{~cm}$

$2330 \pm 70$

UM-1117. 213 to $219 \mathrm{~cm}$

$2660 \pm 80$

UM-1118. 219 to $225 \mathrm{~cm}$

$2420 \pm 65$

UM-1119. 225 to $231 \mathrm{~cm}$

$2735 \pm 75$

\section{Calcrete series}

Calcrete, cryptocrystalline carbonate crusts, 2 to $2.5 \mathrm{~cm}$ thick, resulting from soil-forming processes (Read, 1976), were sampled from Key Largo $\left(25^{\circ} 08^{\prime} \mathrm{N}, 80^{\circ} 21^{\prime} \mathrm{W}\right)$ and Big Pine Key (24 $\left.39^{\prime} \mathrm{N}, 81^{\circ} 21^{\prime} \mathrm{W}\right)$, Florida. Crusts separated into laminae 1 to $4.5 \mathrm{~mm}$ thick. Dated to show that crust forms by orderly deposition of $\mathrm{CaCiO}_{3}$ precipitate from overlying soil layer. Coll and subm 1977 by D Robbin, USGS, Fisher I. Sta, Miami Beach, Floricla.

UM-1079. Key Largo Crust $400 \pm 70$

Lamina 1 to $1.5 \mathrm{~mm}$.

UM-1080. Key Largo Crust $1450 \pm 65$

Lamina 2 to $3 \mathrm{~mm}$.

UM-1081. Key Largo Crust $3100 \pm 80$

Lamina 2 to $4 \mathrm{~mm}$.

UM-1082. Key Largo Crust $4930 \pm 115$

Lamina 2 to $4 \mathrm{~mm}$.

UM-1083. Key Largo Crust

$18,190 \pm 225$

Grain stone underlying laminated crust.

UM-1084. Key Largo Crust

$1685 \pm 80$

Total thickness of laminae without grain stone.

UM-1076. Big Pine Key Crust

Lamina 2 to $4 \mathrm{~mm}$.

$260 \pm 70$ 
UM-1077. Big Pine Key Crust

Lamina 3 to $4 \mathrm{~mm}$.

UM-1078. Big Pine Key Crust

$7900 \pm 190$

Duplicate run of UM-1077.

\section{South Florida coral reef series}

Coral reef accumulation rates in S Florida were studied by dating coral samples (Shinn et al, 1977) from cores taken from Bahia Honda Reef (24 $\left.34^{\prime} \mathrm{N}, 81^{\circ} 20^{\prime} \mathrm{W}\right)$, Carysfort Reef $\left(25^{\circ} 13^{\prime} \mathrm{N}, 80^{\circ} 12^{\prime} \mathrm{W}\right)$, Long Reef $\left(25^{\circ} 27^{\prime} \mathrm{N}, 80^{\circ} 07^{\prime} \mathrm{W}\right)$, and Pulaski Reef (24. $\left.42^{\prime} \mathrm{N}, 82^{\circ} 47^{\prime} \mathrm{W}\right)$. Samples were also obtained from dredge site near Bal Harbor $\left(25^{\circ} 54^{\prime}\right.$ $\left.\mathrm{N}, 80^{\circ} 06^{\prime} \mathrm{W}\right)$ and a sewer trench off Virginia Key $\left(25^{\circ} 44^{\prime} \mathrm{N}, 80^{\circ} 07^{\prime}\right.$ W). Depths reported are from mean sea level. Coll and subm 1975 to 1976 by E Shinn, USGS, Fisher I. Sta, Miami Beach, Florida.

UM-998A. Bahia Honda \#1

$$
6440 \pm 100
$$

Montastrea, $12 \mathrm{~m}$.

UM-998. Bahia Honda

$6170 \pm 80$

Duplicate run of UM-998A.

UM-999. Bahia Honda \# I

$7160 \pm 85$

Montastrea, $13.4 \mathrm{~m}$.

UM-1000. Bahia Honda \#1

$37,480+1300$

Pleistocene coral, $17.1 \mathrm{~m}$.

UM-1001. Bahia Honda \#2

$4735 \pm 85$

Colpophyllia, $10.7 \mathrm{~m}$.

UM-1002. Carysfort Reef \#1

$4570 \pm 85$

Montastrea, $5.8 \mathrm{~m}$.

UM-1003. Carysfort Reef \#1

$5250 \pm 95$

Montastrea, $9.1 \mathrm{~m}$.

UM-1004. Long Reef

$5630 \pm 120$

Montastrea, $8.2 \mathrm{~m}$.

UM-1005. Bal Harbor

$6300 \pm 120$

Siderastrea, $16.1 \mathrm{~m}$.

UM-1006. Pulaski Reef

$5865 \pm 90$

Montastrea, $11.3 \mathrm{~m}$.

UM-1007. Pulaski Reef

$6595 \pm 115$

Montastrea, $13.4 \mathrm{~m}$. 
Diploria, $14.9 \mathrm{~m}$.

UM-1014. Virginia Key

Montastrea Cavernosa, $9.8 \mathrm{~m}$.

\section{Aquifer Recharge series}

Recharge source studies made for sandstone Tamiami Formation underlying Lee and Hendry Co, Florida. The sandstone aquifer is separated from water table aquifer of limestone Caloosahatchee Formation by impermeable green clay layer. Water samples coll from each aquifer encased wells over $42.6 \mathrm{~km}$ extent. $\mathrm{SrCO}_{3}$ was precipitated from water in field. Coll and subm 1977 by T O'Donnell, USGS, Ft Myers, Florida and D Gibbs, U Miami. Comment (TD): samples from sandstone aquifer coll in interval between casing depth and well depth. Water table aquifer samples coll at depths given.

UM-1057. He-529

$14,500 \pm 180$ $47.24 \mathrm{~m}$.

$\left(26^{\circ} 33^{\prime} 10^{\prime \prime} \mathrm{N}, 81^{\circ} 25^{\prime} 09^{\prime \prime} \mathrm{W}\right)$. Casing depth: 41.15m. Well depth:

UM-1058. He-554

$108.3 \pm 1.0 \%$ modern

(26 $\left.36^{\circ} 10^{\prime \prime} \mathrm{N}, 81^{\circ} 25^{\prime} 09^{\prime \prime} \mathrm{W}\right), 3.35 \mathrm{~m}$.

UM-1059. L-2215

$20,230 \pm 280$ $40.54 \mathrm{~m}$.

$\left(26^{\circ} 31^{\prime} 27^{\prime \prime} \mathrm{N}, 81^{\circ} 35^{\prime} 16^{\prime \prime} \mathrm{W}\right)$. Casing depth: 30.18m. Well depth:

UM-1061. L-730

$2200 \pm 75$

$\left(26^{\circ} 31^{\prime} 27^{\prime \prime} \mathrm{N}, 81^{\circ} 35^{\prime} 16^{\prime \prime} \mathrm{W}\right), 5.79 \mathrm{~m}$.

UM-1060. L-2184

$18,840 \pm 370$ $34.14 \mathrm{~m}$.

$\left(26^{\circ} 32^{\prime} 51^{\prime \prime} \mathrm{N}, 81^{\circ} 50^{\prime} 17^{\prime \prime} \mathrm{W}\right)$. Casing depth: $22.86 \mathrm{~m}$. Well depth:

UM-1062. L-1994

$11,710 \pm 125$ $38.10 \mathrm{~m}$.

$\left(26^{\circ} 32^{\prime} 51^{\prime \prime} \mathrm{N}, 81^{\circ} 45^{\prime} 28^{\prime \prime} \mathrm{W}\right)$. Casing depth: $21.34 \mathrm{~m}$. Well depth:

\section{Blanco Trough series}

Benthos gravity core (W7605B-9-GC) recovered from Blanco Trough $\left(44^{\circ} 17^{\prime} \mathrm{N}, 129^{\circ} 39^{\prime} \mathrm{W}\right)$ in $3300 \mathrm{~m}$ water. Dates sedimentation and metal accumulation rates. Coll 1976 by B W Selk, Oregon State Univ, Corvallis, Oregon; subm 1977 by B W Selk and K Rudolph, Univ Miami.

UM-1052. 1 to $9 \mathrm{~cm}$

$14,495 \pm 230$

UM-1053. 12 to $18 \mathrm{~cm}$

$17,200 \pm 140$

UM-1054. 24 to $32 \mathrm{~cm}$

$18,415 \pm 325$

UM-1055. 38 to $46 \mathrm{~cm}$

$24,245 \pm 300$ 
UM-1056. 51 to $59 \mathrm{~cm}$

\section{Black Sea series}

\section{B. Black Sea}

Piston core (P6507-12) from abyssal plain of Black Sea (43 $49^{\prime} 48^{\prime \prime}$ $\left.\mathrm{N}, 35^{\circ} 31^{\prime} 12^{\prime \prime} \mathrm{E}\right)$. Dated to correlate with oxygen isotope record from core. Coll 1965 by RSMAS, Miami; subm 1977 by J Southam and M Boehm, RSMAS, Miami.

\section{UM-1064. 53 to $69 \mathrm{~cm}$ \\ UM-1065. 145 to $153 \mathrm{~cm}$ \\ UM-1066. 163 to $172 \mathrm{~cm}$ \\ UM-1068. 645 to $655 \mathrm{~cm}$}

$15,815 \pm 450$

$12,190 \pm 145$

$16,600 \pm 400$

$>34,600$

\section{Carrie Bow series}

\section{Belize}

Coral from core taken near Carrie Bow Cay, Belize $\left(16^{\circ} 50^{\prime} \mathrm{N}\right.$, $\left.88^{\circ} 05^{\prime} \mathrm{W}\right)$. Dated to study reef accumulation rates. Depth from top of core. Coll and subm by E Shinn.

UM-1009. Carrie Bow \#2

$5625 \pm 85$

Montastrea, depth $5.80 \mathrm{~m}$.

UM-1010. Carrie Bow \#2

$6165 \pm 90$

Porites, depth $8.20 \mathrm{~m}$.

UM-1011. Carrie Bow \#2

$6140 \pm 90$

Porites, depth 11.0m.

UM-1012. Carrie Bow \#2

$7175 \pm 100$

Porites, depth $17.70 \mathrm{~m}$.

UM-1013. Carrie Bow \#4

$6960 \pm 110$

Montastrea, depth $15.50 \mathrm{~m}$.

REFERENCES

Davis, J H, 1943, The natural features of southern Florida: Especially the vegetation, and the Everglades: State of Florida Dept of Conservation. Geol Bull 25, Tallahassee, Florida, $311 \mathrm{p}$.

Piepgras, D and Stipp, J J, 1977, University of Miami radiocarbon dates VIII: Radiocarbon, v 19, p 118-126.

Read, J F, 1976, Calcretes and their distinction from Stromatolites: Stromatolites, New York, Elsevier Pub Co, 438 p.

Shinn, E A, Hudson, J H, Halley, R B, and Lidz, B, 1977, Topographic control and accumulation rate of some Holocene coral reefs: South Florida and Dry Tortugas, 3rd Internatl coral reef symposium, Proc, Miami, Florida, Gcology, v 2, 628 p.

Stipp, J J, Eldridge, K L, and Cadwell, R, 1976, University of Miami radiocarbon dates VI: Radiocarbon, v 18, p 210-220.

Stipp, J J, Eldridge, K L, and Valenziano, K, 1976, University of Miami radiocarbon dates VII: Radiocarbon, v 18, p 371-375. 